\title{
In silico prioritization for endocrine active substances (EAS) and their in vitro validation
}

\author{
Ivano Eberini ${ }^{1}$, Luca Palazzolo ${ }^{1,2}$, Ad A.C.M. Peijnenburg ${ }^{3}$, Uliano Guerrini ${ }^{1}$, Chiara Parravicini ${ }^{1}$, Angelo Moretto ${ }^{2}$, and Toine \\ F.H. Bovee ${ }^{3}$ \\ ${ }^{1}$ Dipartimento di Scienze Farmacologiche e Biomolecolari and 2Dipartimento di Scienze Biomediche e Cliniche "L. Sacco", \\ Università degli Studi di Milano, Milano, Italy; ${ }^{3}$ RIKILT-Institute of Food Safety, Wageningen University, Wageningen, the \\ Netherlands
}

In silico molecular docking can be a cheap and fast strategy to estimate the binding free energies, and consequently the dissociation constants, for a set of compounds with respect to their putative targets. Interesting targets for EAS are the ligand binding domains (LBD) of the human nuclear receptors for the sex hormones, i.e. the estrogen, androgen, progesterone, and (gluco)corticoid receptor.

The Horizon 2020 project EuroMix (http://euromixproject.eu) aims to establish and disseminate new, efficient and validated strategies for the risk assessment of mixtures, while limiting the use animal tests. This presentation deals with a part of EuroMix that is intended to set up a testing approach for mixtures of endocrine disrupting chemicals, focusing on estrogenic and anti-androgenic effects. For that purpose, a combined adverse outcome pathway (AOP) was constructed, including molecular initiating events, key events, and adverse outcome (reproductive dysfunction). Using this combined AOP as framework, cognate in silico and in vitro tools as well as the in vivo confirmation studies were selected, i.e. in silico: h-ER and h-AR docking; in vitro: cell-based ER and AR transcrip- tional activation bioassays and the H295R steroidogenesis assay; and in vivo: the fish sexual development test (FSDT, OECD Test No. 234) and a rat study, examining in (male) offspring a num- ber of parameters, such as anogenital distance, cryptorchidism, and nipple retention.

Preliminary in silico and in vitro tests of selected reference chem- icals show for individually tested compounds a great correlation between the in silico determined binding energies and the in vitro measured hormonal activities. 